\title{
On the Methods for Detecting Brain Tumor from MRI images
}

\author{
Aswani K, Menaka. D., Manoj M K.
}

\begin{abstract}
Brain tumor detection from MRI images is a challenging process due to high diversity in the tumor pixels of different peoples. Automatic detection has got wide spread acclaim because the manual detection by experts is time consuming and prone to error in judgment. Due to its high mortality rate, detection of tumor automatically is a new emerging technique in bio medical imaging. Here we present a review of few methods from simple thresholding to advanced deep learning methods for segmentation of tumor from MRI data. The segmentation of tumor methods is classified to image segmentation using gray level processing, machine learning and deep learning. The results of various methods are compared to find the best methods available. As medical imaging methods have improving day by day this review will help to understand emerging trends in brain tumor detection.
\end{abstract}

Keywords: MRI, Segmentation, Machine Learning, Deep Learning

\section{INTRODUCTION}

Brain tumor is a collection of abnormal cells formed inside the brain. Magnetic Resonance Imaging (MRI) is used by the medical practitioners to detect and diagnose brain tumor to different categories. For example, the tumor can be cancerous (Malignant) or non-cancerous (Benign). Cancerous tumor is further divided to High Grade Gliomas (HGG) and Low Grade Gliomas (LGG) based on their mortality rate. Tumor detection from MRI is done by expert medical professionals. Manual detection is prone to error due to the diverse nature of the tumor. So automatic detection became important and this study explores many methods used for detection and segmentation of tumor from MRI images. Automatic detection of tumor start from simple thresholding and developed to various sophisticated methods like deep learning. The methods are classified in to three categories. Firstly the image segmentation through image processing by various methods. Then Machine Learning (ML) with various feature extraction methods and finally the Deep Learning (DL) methods. All of these methods have their own advantages and disadvantages. The selection of the method is based on the problem selected. Usually in medical diagnostics the importance is given to accuracy in the segmentation rather than the speed of operation. Here DL have clear advantage

Revised Manuscript Received on July 05, 2020.

* Correspondence Author

Aswani K*, Dept. of Electronics and Communication, Noorul Islam Center for Higher Education, Thuckalay, India. Email: aswaninarayanan.k88@gmail.com

Dr. D. Menaka, Dept. of Electronics and Instrumentation, Noorul Islam Center for Higher Education, Thuckalay, India. Email: menakaberita@gmail.com

Manoj M K, Dept. of Electronics and Communication, MEA Engineering College, Perinthalmanna, India. Email: manojmk@meaec.edu.in over the other methods. The segmentation through image processing is the simple image processing operations on the pixel values of the MRI like thresholding. Various methods for segmenting the required portion from an MRI is developed over the years. Some of which are Watershed algorithm, Genetic algorithm, Fuzzy Clustering, Deformation model, Markov Random Field etc. All the above method have the advantages like speed of operation which is useful if fast results are required. But they clearly lack the performance when there is pixel intensity variations, diverse nature of the tumor, machine and other noise effects. To overcome the deficiencies specified above special features are extracted from the MRI belong to the tumor and other parts. These features are then used to train a classifier which can predict the tumor pixel by pixel. The method is generally called Machine Learning. In our study it is shown that some of the methods which segment the tumor accurately compared to the general image processing techniques. Some of the methods are Support Vector Machine (SVM), Random Forest (RF), and Naïve Bayesian (NB), K nearest Neighbor (KNN) and Artificial Neural Networks (ANN). All the methods above require special features separating a tumor pixel from Non tumor pixel. The accuracy of the methods depend on the feature value and the number of features extracted. Various methods are available for extracting low level and high level features from the MRI images. Since the classifiers need to be trained before classification large number of data is required. The database can be collected from hospitals, or there are publically available database for tumor detection challenges. One of the database is BRATS. The database have different versions starting from 2012. Using the same database for different methods make the comparison study effective. So most of the methods discussed here use the latest BRATS database for training and testing. Deep Learning does not require the features for training and testing. The Features are extracted from the various levels involved in the layers. So it make the method user friendly but the complex nature of designing the deep layers ensure the expertise required in the field. Though many deep methods are available it is clear that the tumor detection using Convolutional Neural Network $(\mathrm{CNN})$ and Generative Adversarial Network (GAN) are particularly interesting because of their ability to segment tumor pixel from others. Like ML a huge database is required for training the network. The training process in CNN and GAN are both complex and time consuming but the accuracy in segmentation of the methods outperform all the ML methods discussed above.

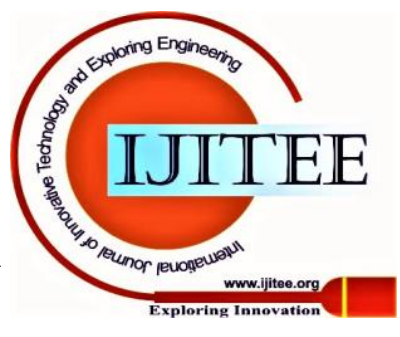


The reminder of the paper is organizes as follows. First we discuss the image preprocessing and the BRATS database used for tumor detection. Then in the second section various methods in the image segmentation is studied and their weakness is identified. In third section the machine learning methods with different feature extraction techniques. Fourth section discusses the deep learning in detail. The concentration is given mainly on CNN and GAN. Finally the methods discussed so far are compared and list out the advantages and disadvantages. This will lead to understand the current research and trends going on in the field of brain tumor segmentation from MRI images.

\section{IMAGE PREPROCESSING}

MRI images are prone to intensity variations due to bias field distortion. The image obtained of a patient can vary if the same is taken at a different time. Although many intensity normalizations methods are available it is found that the method suggested in in [1] and proposed by [2] are effective. Here a set of intensity landmarks $\left\{p c 1, i p_{10}, i p_{20} \ldots i p_{90}, p c 2\right\}$ are learned from a set of training sequences. Here $i p_{10}$ represent the $l^{\text {th }}$ percentile between $p c l$ and $p c 2$.During the preprocessing stage all the intensities between two landmarks are linearly mapped to one of the landmark. In this way a histogram of images of same subjects have some similarity. After the preprocessing the normalization also done for zero mean unit variance images. In addition to normalization sometimes contrast starching is also necessary for deep learning based tumor segmentation. The idea is to stretch the tumor pixels from other parts of the image increase the learning efficiency of the neural network. Most of the methods discussed here use the BRATS database for training and testing of tumor detection challenges. BRATS database is maintained by the Section for Biomedical Image Analysis (SBIA) of Perelman school of medicine, University of Pennsylvania. The database is useful due to its popularity and can be used to compare different studies in the field of Brain tumor segmentation

\section{IMAGE SEGMENTATION METHODS}

In this section an overview of various image processing techniques to segment a portion of the image is given. In [3], [5] authors suggest a simple thresholding called Otsu threshold to detect the tumor. Otsu threshold use the histogram of the image to find out a single threshold which separate the pixels to two classes. A multilevel thresholding method is used in [4], [6] to find the optimal segmentation. The multi-level threshold is derived from a fuzzy set. A level set algorithm is further employed to detect the tumor. Thresholding only to segment the tumor has many disadvantages like unwanted portions will be detected as tumor when a single threshold is used, even though denoising is applied in many methods it will not be sufficient to avoid pixel variations due to noise and leads to inaccurate detection.

The inaccuracy of simple thresholding lead to more sophisticated segmentation methods. In [7] the authors provide Fuzzy C-means to cluster the data points or pixels to a particular membership function.an objective function is used to associate a data point to a membership function. $\mathrm{K}$ means clustering [8] is another popular method for segmenting the image. In this method two cluster centers are selected based on a heuristic way. The pixel which have minimum distance from the cluster centers are grouped together. Then the cluster centers are recomposed by averaging the pixels in the group. The process is repeated until the best cluster centers are formed. Hidden Markov Random Field [HMRF] is another method [9] in segmentation of image. HMRF is a graphical probability model in which the true states are not observed but are estimated indirectly through an observation field. In a HMRF model, there is an observable random field $\mathrm{y}=$ $\left(y_{1} \ldots . . y_{N}\right)$ where each $y_{i}$ is the feature value of a pixel. The goal is to infer a hidden random field $x=\left(x_{1} \ldots \ldots x_{N}\right)$. In the image segmentation context, $\mathrm{x}_{\mathrm{i}}$ is a configuration of labels and $\mathrm{L}$ is the set of all possible labels. In [10] the Fuzzy C-means based genetic algorithm is used for tumor detection. The initial cluster is found using Fuzzy C- means then it is optimized using the genetic algorithm. The approach has a series of operations like mutation, cross over etc. mimicking the human population evolution to find the best possible cluster among the many.

\section{MACHINE LEARNING METHODS}

The most used machine learning classifier for tumor detection is SVM classifier. SVM is a supervised learning model that performs well on two class problems. It uses a set of training labeled samples to make model which separates one class from another making a non-probabilistic binary classifier. In [11] authors suggest SVM classifier for brain tumor segmentation. They apply anisotropic filtering for noise reduction in the dataset. The dataset is then labeled as tumor and non-tumor pixels and given to the classifier for training. Some post processing operations are necessary for final segmentation. The method generally have less accuracy because of directly labeling the intensity level to labelling. For better performance feature extractions from the data set is required. Wavelet transforms used in [12] to find some specific features from the dataset used for training. Here authors initially perform a segmentation using thresholding [3]. The result is then demised using anisotropic filtering [11]. Then special features are extracted using wavelet transform. The low level and high level spatial features are stored and used for training the SVM classifier. The authors claim an accuracy of $86 \%$ while segmenting the tumor from an image.

Another method [13] is a combined method which uses genetic algorithm with SVM for segmentation. Here the dataset is preprocessed using several morphological operations like filtering, edge detection, opening, closing etc. to extract the tumor portion from the dataset. Then features like shape, texture, intensity are obtained from the tumor portions. A total of 14 features are extracted from the entire dataset. The features are optimized using the genetic algorithm. The optimized features then applied to training and testing tumor segmentation. The authors claims to have high accuracy compared to non-optimized features using SVM.

In [14] deep leaning CNN is combined with SVM for better performance. In the first stage, the authors trained $\mathrm{CNN}$ to learn the mapping from the image space to the tumor label space.

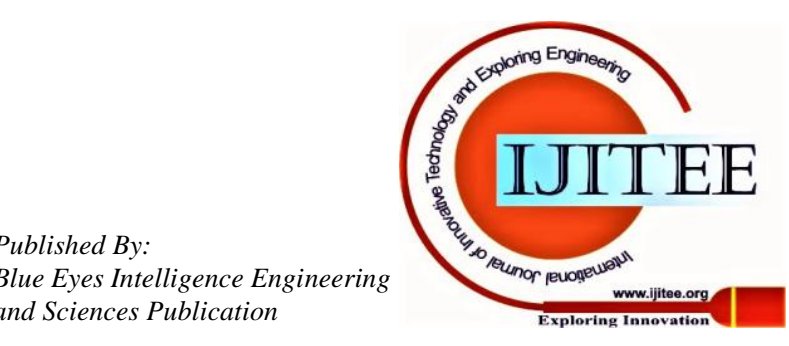


During the testing phase, they used the predicted label output from $\mathrm{CNN}$ and sent it along with the testing image to an SVM classifier for accurate segmentation. Since $\mathrm{CNN}$ work on image patches the output of CNN is treated as a local stage and the obtained result is used to train a SVM classifier for better performance. The comparison results shows the combined method have better classification of tumor pixel from their standalone results.

Another combination is done in [15] where feature set is reduced using rough $\mathrm{K}$ means algorithm. A total of 12 features are extracted from the dataset. The feature like Entropy, Homogeneity, Energy, Contrast etc. makes the feature set a large one. So $\mathrm{K}$ means is employed to group the features to make a reduced feature set. This reduced set is used for training and testing the SVM classifier. The comparison with other combined methods is also done and the results clearly shows the advantage of the method proposed by the authors.

Another popular machine learning classifier is KNN. In [16] the authors compare the performance of KNN over SVM. Gray Level Co-occurrence Matrix (GLCM) of the dataset is used as features for training and testing. GLCM is a calculation of how often different combination of gray levels occur in an image. It is very useful in texture analysis. The results show that KNN produce comparable performance with SVM. Another method [17] use KNN for 3d tumor segmentation. Here the features are manually collected from the voxels. A $6 \mathrm{D}$ dataset is made from the training samples available. The authors used BRATS database for training and testing. The features are used for training the KNN classifier.

In [18] authors compared the performance of KNN over Fuzzy $\mathrm{C}$-means and $\mathrm{K}$ means clustering. Again the authors used GLCM as features. Several preprocessing operations are performed before finding the features from the dataset. Here the authors show that Fuzzy C-means has a better accuracy in segmentation compared to $\mathrm{KNN}$ or $\mathrm{K}$ means. A hybrid form of SVM and KNN is used in [19]. Here the authors performed a skull masking to remove the boundary of the brain skull part. Then several first and second moment features are extracted from the training dataset. Entropy, Dissimilarity, Contrast are some of these features. A total of six features along with symmetrical feature is used for training. In this hybrid method first the feature set is trained with an improved SVM classifier which combines SVM with KNN.

The results are compared with normal SVM and KNN and got better results than both. The comparison parameters used are Accuracy, Sensitivity, and Specificity.

Region growing segmentation along with KNN is proposed in [20]. Here the image is preprocessed before given to feature extraction. The co-occurrence matrix is developed from the training set. Then from the co-occurrence matrix seven parameters are computed. The parameters are second angular moment, correlation, moment difference, inverse difference moment, dissimilarity, entropy and maximum probability. To perform multi resolution analysis a wavelet decomposition is also performed. The analysis of the multi-resolution medical image consists of decomposing the original space into four spaces to allow calculation of mean energy of each channel for three level wavelet decomposition. In the results both tumor and healthy tissues parameter values are shown which ensure a good comparison result. The trained KNN classifier is used to separate healthy tissue from tumor one. The authors conclude the method is good for texture analysis performed in hospitals.

Naïve Bayesian classification (NBC) is another technique for tumor segmentation. It is based on Bayer's theorem. In simple terms a naïve Bayesian classifier assumes that the presence of a feature in a particular class is unrelated to the presence of any other features. It can be used for multi class classification even though tumor detection need only binary classification. In [21] authors use NBC for tumor classification. The dataset for training is denoised using a median filter. GLCM is used for textural pattern analysis. From the GLCM matrix many features like Entropy, Correlation, and Energy etc. are evaluated for training the classifier. Instead of tumor segmentation the authors predict the condition of the tumor. A three level classification is done depending on the condition of the tumor namely Normal, Benign, and Malignant. Here malignant shows the serious medical condition while normal is MRI with non-tumor pixels and benign stands between these two. The authors claim high accuracy in tumor classification. Though NBC is used for tumor classification it can also be used for tumor segmentation but the works done in that area seems less than other machine learning methods.

The random Forest is another classification algorithm in machine learning. It consists of many decision tress. The algorithm used bagging and feature randomness to determine each individual trees and create an uncorrelated forest of trees which can classify or segment the tumors in a significant way. [22] Is such a work where the features are extracted from a fractal based brain tissue. The intensity of the tumor pixels are labeled from other tissues and applied to the $\mathrm{RF}$ classifier for training. The result obtained is compared with a modified adaboost algorithm and found better performance than the latter. A cascaded RF classifier is used in [23] for tumor segmentation. The authors used BRATS2013 dataset for training and testing the performance of the algorithm. After preprocessing the dataset for normalization features are extracted. Here authors took textual, intensity and contextual information as features along with feature histograms of two hundred candidates. The authors found RF classifier is useful because of the following properties. RF classifier's ability to parallelize the process being an ensemble of decision trees; randomness during the training for generalization of unseen instances; its ability to deal with high dimensional features; and its consistency for handling multiple class label problems. The results re then compared with manual segmentation as well as other well-known machine learning methods.

Concatenated and connected RF is used in [24] to detect the brain tumor from MRI images. The authors introduce a new methodology for concatenating RF with contour model to detect the Gliomas from MRI images. The authors employ a feature representations learning strategy to effectively explore both local and contextual information from multimodal images for tissue segmentation by using modality specific random forests as the feature learning kernels. Different levels of the structural information is subsequently integrated into concatenated and connected random forests for gliomas structure inferring.

Published By:

Blue Eyes Intelligence Engineering and Sciences Publication 
Finally, a novel multiscale patch driven active contour model is exploited to refine the inferred structure by taking advantage of sparse representation techniques. Competitive accuracy is obtained by comparing with the well-known methods as claimed by the authors.

Artificial neural network (ANN) is another well-known method for segment the tumor from MRI images. A Self Organizing Map (SOM) is type of ANN in which training is done in an unsupervised way to reduce the dimension of the training samples called a map. So the method is typically a dimension reduction technique. SOM is different from other ANN methods because they apply a competitive learning opposed to error correction learning while preserving the topology of the input space. In papers [25] and [30] authors use SOM to segment the tumor part from non-tumor part of a MRI image. Brats 2015 dataset is used for training and testing the method. GLCM is used to get the textural information of the dataset. From the GLCM matrix a total of seven features are extracted. There is more than 200 candidate image which make the feature space enough for training. Two different topology is applied for SOM unsupervised learning named Rectangular and hexagonal. The result shows good performance and is comparable to any supervised learning methods.

ANN is used for tumor type classification in [26], [29]. Here the authors propose a simple ANN to classify the tumor as Malignant or benign. In the training stage the images are down converted to low level and high level images using Discrete Wavelet Transform (DWT). Fuzzy C means is then employed for clustering the images. The clustered images are employed for feature extraction. Several statistical features are extracted by known methods along with gabor wavelet features. There features are used to train a simple ANN for pattern recognition.

A comparison of SVM and ANN is employed in [27] for tumor classification. The method has four main fields. First the tumors are segmented from the training images using an active contour model. Then GLCM based features are extracted. Since feature space is very large optimization and features are classified using ANN and SVM and their results are compared. The authors claim that ANN with GA outperform SVM with GA in terms of accuracy.

A simple Principle Component Analysis (PCA) based tumor classification is performed in [28]. Here initially gradient vector flow is used to segment the tumor area from training images. Features are extracted using PCA. A total of 208 features are extracted from each image. Depending on the training set a huge feature set is developed. Feature reduction is performed using Singular value decomposition (SVD). The features are used for training ANN for pattern recognition and further for tumor classification.

A summary of machine learning methods is given here. All the methods depend on the features extracted and number of features. Intensity based features poses a problem for all methods as the tumor intensity is diverse among the dataset. Special features like textural information (GLCM) is used by most of the methods for segmentation and classification. Among the classifiers SVM and ANN perform better for tumor segmentation while for classification almost all the methods provide the same accuracy. The accuracy presented by most of the methods are very high but still less for medical imaging particularly for tumor segmentation. reduction is performed using genetic algorithm. Finally the

Deep learning methods provide an alternative way as it removes the need for feature detection from dataset and provide very high accuracy compared most of the state of art machine learning methods.

\section{DEEP LEARNING METHODS}

Deep learning is a trending subject nowadays. Many deep architectures are available which are used for many image processing applications like segmentation and classification. In the context of brain tumor segmentation two methods which are particularly suitable also have very high accuracy compared to other methods are segmentation using CNN and GAN.

In [1] the authors present the Deep Learning CNN for the segmentation of tumor from MRI images of brain. The training images are preprocessed as explained in section II of this paper. To increase the training set of the database data augmentation is performed. Image rotation is preferred by the authors to make different data from available dataset. Augmentation may come as a necessity in image segmentation due to the difficulty in getting medical data for public usage. The main layers of a CNN is described below.

The convolution layer is the backbone of $\mathrm{CNN}$ along with back propagation as training algorithm. In the convolution layer a signal or an image is convolved with filter kernels to obtain the feature maps. Back propagation is used to update the filter weights while training the network. In CNN the image in training set is divided in to patches and convolution operations performed on these. This makes CNN have lesser weights to train compared to the Fully Connected (FC) layers. Since the kernel s shared among all the images same feature is obtained for similar patches independent of the location. The next step after convolution is usually a nonlinear activation function. The activation function is responsible for which data at the output of convolutional layer is passed to the next layer. To avoid the vanishing gradient problem the

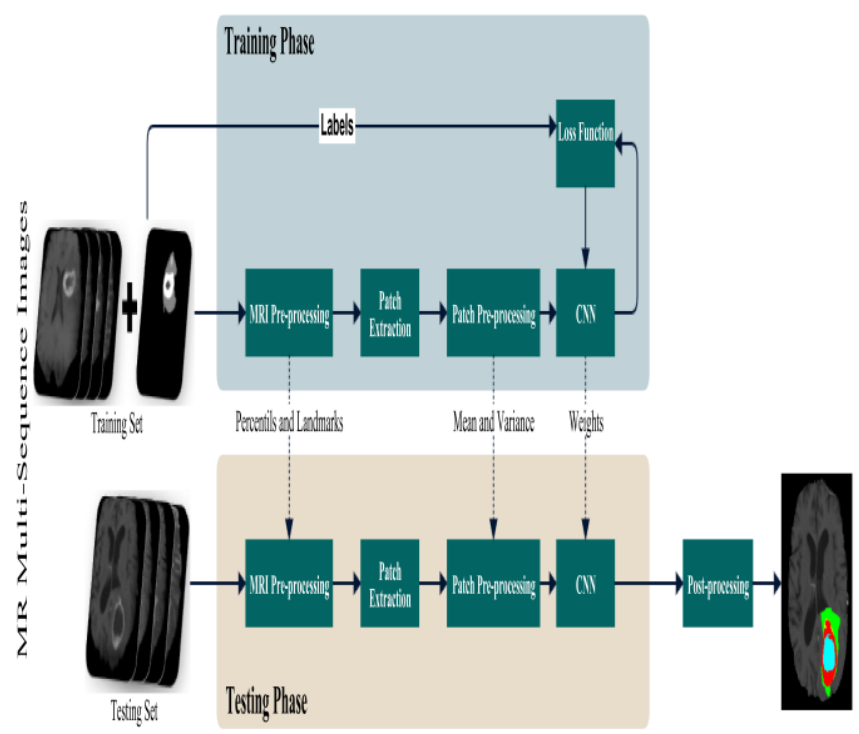

Fig. 1. CNN architecture

Published By:

Blue Eyes Intelligence Engineering 
authors used ReLu layer instead of the commonly known sigmoid, hyperbolic tangent etc. The selection ReLu also speed up the training process. A leaky ReLu is also suggested because imposing a zero in ReLu may impair the flow of gradient sometimes. The next step involved in the process is pooling. Pooling combines the spatially related features thus reducing the dataset and computational burden. It also speed up the training process and removes insignificant data in the feature maps. Two methods are available for pooling the features namely average pooling and max polling. Regularization is also performed while training. This is equivalent to drop out layer present in the FC layers. The usage of drop our layers reduces over fitting because it drops the features which are insignificant to the training process on each iteration.For image an image of size 256x256 the authors used six convolutional layer, two max polling layer and three fully connected layers for their work. Categorical cross entropy is used as loss function for training the network through back propagation. The loss function is minimized in each iteration. The authors used BRATS2015 database and extracted around 450000 patched from the trainings set since $\mathrm{CNN}$ operated on the patches. The performance of the segmentation is measured using the following three metrics. The Dice Similarity Coefficient (DSC), Sensitivity, and Positive Predictive value (PPV).Both HGG and LGG are tested in the algorithm and the results obtained shows the advantage of deep learning over machine learning methods. The authors [1] participated in BRATS challenge for tumor detection and segmentation and obtained first place in BRATS2013 database challenge and second place in BRATS2015 database challenge. This ensure the advantage of using $\mathrm{CNN}$ in tumor segmentation in MRI images. The training and testing network used by the authors is shown in Fig1 for reference.Fig1. CNN architecture

In [29, 33-37] authors present a multichannel input $\mathrm{CNN}$ for feature extraction using CNN. Instead of giving the patches to input layer the authors find the super pixel segmentation of the image first in view of saliency detection then each super pixel is applied to a different $\mathrm{CNN}$ architecture. Each CNN architecture generate hierarchical features and pooled to combine the final feature set. The authors presented two experimental $\mathrm{CNN}$ architecture for multichannel input namely Xception model and Dense Net model. High feature recognition rate is obtained as claimed by the authors. A cascaded CNN is presented in [30] for gliomas detection from MRI images. Two different features are extracted namely the Gaussian-pyramid multiscale input features-fusion technique and the brainstem-region feature enhancement. CNN feature along with SVM classifier is used for segmentation of the gliomas. Gaussian-pyramid multiscale input feature fusion is added to our gliomas-segmentation task to solve the problems of size variety and weak brainstem-gliomas boundaries.

Generative Adversarial Network is a machine learning method developed by Ian Goodfellow and his friends in 2014. It is developed as an unsupervised learning technique that is given a training set it can generate a new data statistically similar to the original one. For example GAN trained on images can regenerate images which have realistic characteristics. Though developed as an unsupervised learning method GAN is now used for semi supervised and supervised learning such as tumor segmentation training and classification. GAN finds its use in many areas particularly in art, fashion, advertising, science and video games. GAN is useful for generating synthetic dataset as an alternative to MRI images. Since medical image data availability is very low generating synthetic dataset resembling characteristics similar to MRI is very useful in the field of medical diagnostics. In [31] the authors present such a network for data generation and RESNET50 is used for tumor detection. Two methods are used by the authors for the generation of dataset named as Progressive Growing of GANs (PGGAN) and Multimodal Unsupervised Image-to-image Translation (MUNIT). The first method is a multistage noise to image generation GAN which generate 256x256 high resolution MRI images while in the latter method GAN is combined with auto encoders called SimGAN which refines the shape/texture of the images generated by PGGAN. PGGAN is a GAN training method which progressively grows a generator and a discriminator. The PGGAN architecture adopts the Wasserstein loss with gradient penalty and generate an image of size $256 \times 256$. SimGAN is then used to refine the generated image and fed to the RESNET50 for tumor segmentation. RESNET50 has CNN at its background and use back propagation for training. In [32] authors present the segmentation of tumor from 3D image data. The method proposed is Multi channel CNN with conditional random field. After the preprocessing of images coarse segmentation is performed on the image patches. The three view of slices named axial, coronal and sagittal are used for segmentation. A two channel CNN (TTCNN) is used for finding the tumor part. The contour of gliomas are obtained from this stage. Conditional random field is used for fine segmentation of the tumor images.

\section{COMPARISON OF DIFFERENT METHODS}

Here the comparison of different methods discussed so far are compared and their outcomes are discussed. Most of the method use the BRATS dataset for training and testing. The efficiency and limitation of the each method is also discussed. The methods which use BRATS dataset have more than 500 images available with Low and High grade Gliomas.

Table- I: Currently Developed Filters/Architecture and Training/Efficiency/Limitation

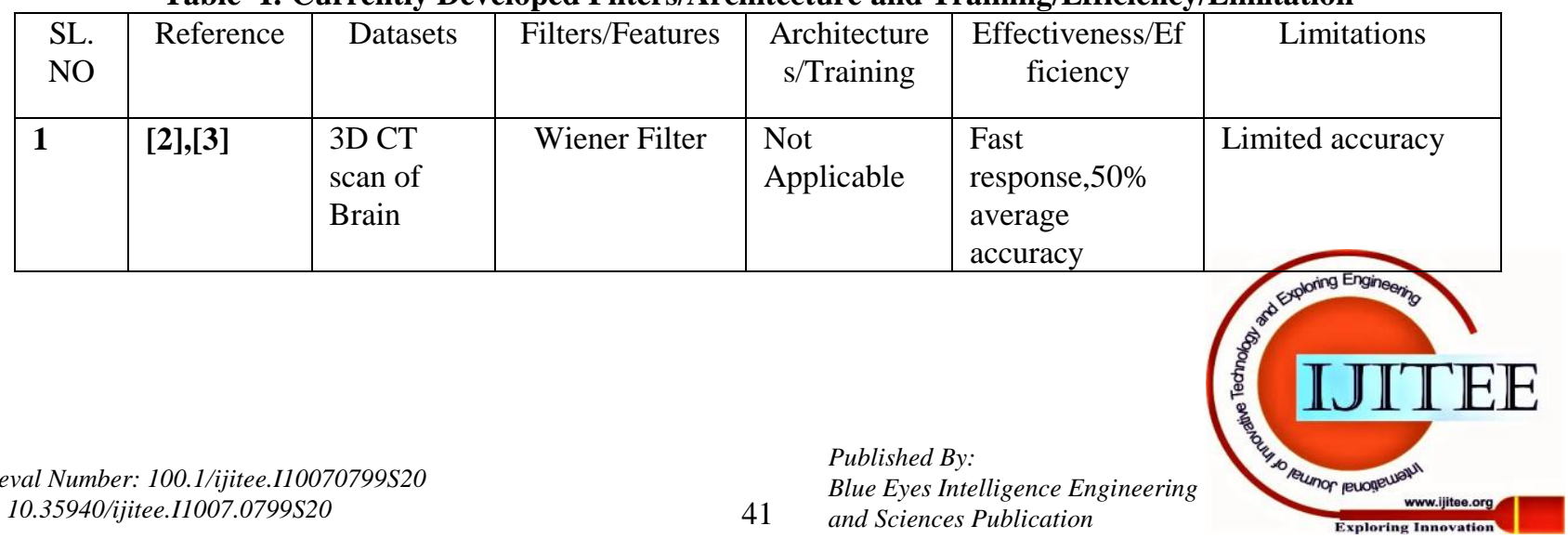


On the Methods for Detecting Brain Tumor from MRI images

\begin{tabular}{|c|c|c|c|c|c|c|}
\hline 2 & {$[4],[5]$} & $\begin{array}{l}\text { BRATS } \\
\text { dataset }\end{array}$ & $\begin{array}{l}\text { Medain } \\
\text { Filtering,Histogr } \\
\text { am Equaliztion }\end{array}$ & $\begin{array}{l}\text { Not } \\
\text { Applicable }\end{array}$ & $\begin{array}{l}53 \% \text { Similarity } \\
\text { index and } 73 \% \\
\text { Jaccard index }\end{array}$ & $\begin{array}{l}\text { Illumination } \\
\text { variation among the } \\
\text { dataset affect the } \\
\text { accuracy }\end{array}$ \\
\hline 3 & [6] & $\begin{array}{l}2 \text { D CT } \\
\text { scan } \\
\text { Dataset of } \\
\text { Brain }\end{array}$ & Wavelet filtering & $\begin{array}{l}\text { Not } \\
\text { Applicable }\end{array}$ & $\begin{array}{l}\text { More robust due } \\
\text { to adaptive } \\
\text { thresholding } \\
\text { calculation }\end{array}$ & $\begin{array}{l}\text { Illumination from } \\
\text { hard tissues affect } \\
\text { segmentation }\end{array}$ \\
\hline 4 & {$[7],[11]-16$} & $\begin{array}{l}\text { BRATS20 } \\
\text { 15,BRATS } \\
2013, \text { CT } \\
\text { scan of } \\
\text { Brain }\end{array}$ & $\begin{array}{l}\text { Wavelet, } \\
\text { Anisotropic, } \\
\text { HoG, Gabor, } \\
\text { Median, } \\
\text { gaussian }\end{array}$ & $\begin{array}{l}\text { Fuzzy } \\
\text { C-Means, } \\
\text { KNN } \\
\text { Wavelet } \\
\text {,PSO with } \\
\text { SVM }\end{array}$ & $\begin{array}{l}90 \% \text { Average } \\
\text { validation } \\
\text { accuracy with } \\
\text { SVM training } \\
\text { and validation }\end{array}$ & $\begin{array}{l}\text { Frequent patterns } \\
\text { are limited. } \\
\text { Accuracy not up to } \\
\text { medical standards. }\end{array}$ \\
\hline 5 & [17]-[20] & $\begin{array}{l}\text { BRATS20 } \\
13\end{array}$ & $\begin{array}{l}\text { Dilation, } \\
\text { Erosion, Gray } \\
\text { scale features, } \\
\text { Wavelet Filters }\end{array}$ & $\begin{array}{l}\text { KNN/Hybrid } \\
\text { KNN-SVM, } \\
\text { FCM }\end{array}$ & $\begin{array}{l}\text { Hybrid } \\
\text { classifiers } \\
\text { achieve } 98 \% \\
\text { accuracy }\end{array}$ & $\begin{array}{l}\text { Most of the method } \\
\text { require skull } \\
\text { masking and } \\
\text { training time } \\
\text { required is high }\end{array}$ \\
\hline 6 & {$[22]-[25]$} & $\begin{array}{l}\text { 2D CT } \\
\text { scan, } \\
\text { BRATS } \\
2017\end{array}$ & $\begin{array}{l}\text { LMMSE filter, } \\
\text { GLCM features }\end{array}$ & $\begin{array}{l}\text { Random } \\
\text { Forest with } \\
\text { Active } \\
\text { Contour }\end{array}$ & $\begin{array}{l}\text { Brings more } \\
\text { accuracy and } \\
\text { robustness } \\
\text { through shape } \\
\text { priori and spatial } \\
\text { constraint } \\
\text { scheme }\end{array}$ & $\begin{array}{l}\text { The features are not } \\
\text { optimal, Large } \\
\text { training data } \\
\text { requirement, } \\
\text { Ground truth labels } \\
\text { are biased using } \\
\text { algorithm }\end{array}$ \\
\hline 7 & {$[26]-[28]$} & $\begin{array}{l}\text { PGIMER, } \\
\text { SPL }\end{array}$ & $\begin{array}{l}\text { Statistical, } \\
\text { Gabor filter, } \\
\text { PCA, Genetic } \\
\text { Algorithm }\end{array}$ & $\begin{array}{l}\text { Feed } \\
\text { Forward } \\
\text { ANN with } \\
\text { Back } \\
\text { propagation }\end{array}$ & $\begin{array}{l}\text { GA with ANN } \\
\text { has accuracy in } \\
\text { the range } 75.6 \% \\
\text { to } 94.9 \%\end{array}$ & $\begin{array}{l}\text { Speed of operation } \\
\text { is less compared to } \\
\text { GA with SVM }\end{array}$ \\
\hline 8 & $\begin{array}{l}{[1],[29],[3} \\
3-37]\end{array}$ & $\begin{array}{l}\text { BRATS20 } \\
15,2017\end{array}$ & $\begin{array}{l}\text { Fully } \\
\text { convolutional } \\
\text { neural networks }\end{array}$ & $\begin{array}{l}\text { Stochastic } \\
\text { gradient } \\
\text { descent, } \\
\text { adaptive } \\
\text { moment } \\
\text { estimation }\end{array}$ & $\begin{array}{l}\text { Achieved } \\
\text { computational } \\
\text { efficiency }\end{array}$ & $\begin{array}{l}\text { Relationship among } \\
\text { image patches is } \\
\text { typically } \\
\text { lost }\end{array}$ \\
\hline
\end{tabular}

\section{CONCLUSION}

The purpose of this work is to provide an overview of various tumor segmentation methods. Most of the methods cluster the tumor using different features and spatial information from the neighbor of MRI images. The aim of all these help the physician for early judgment of tumor and develop the treatment procedure for the affected patients.

Most of the tumor segmentation algorithms works well for researchers but seldom used in clinical process. This is due to the fundamental difference in approach of the researchers and clinical persons. The clinical persons lacks knowledge in existing tumor segmentation software. This often leads to manual detection of tumor and may prone to error. So a bridge is necessary between the manual segmentation and the automatic tumor segmentation methods. Nowadays some clinical process are using softwares for the early diagnosis of tumor. Apart from the evaluation of tumor by software processing the running time to detect tumor is also important in clinical process. For example in the paper it is shown that the deep learning method show the better evaluation statistics but the running time required to process an image for tumor detection is almost over a few minutes. This is unacceptable for clinical procedures. Even though real time detection is not practical methods should be developed to run the algorithm faster. Another aspect the clinical persons worry about automatic segmentation is their robustness. A software which work well initially may fail after time progresses. The diversity of the tumor is the fundamental reason for this behavior. So the training algorithm of tumor should be performed on a time basis to eradicate this problem. Methods which are providing good robustness and reasonable running time are on the development stage by the companies working in the bio medical research field.

Before deep learning most of the tumor segmentation algorithms relied on the machine learning methods. Most of the machine learning methods depends on the features extracted from the tumor images. Because of the diverse nature of the tumor many relevant and meaningful features have to be developed further for better understanding of tumor pixel from others. Deep learning is developed later for tumor detection and segmentation purposes. Initially they are used only for classification tumor to different grades. For example a $\mathrm{CNN}$ can be trained for classifying the tumor to LGG or HGG. But for classification machine learning methods are enough.

\section{Published By:}

Blue Eyes Intelligence Engineering 
Most of the machine learning method discussed above like SVM, KNN are well suited for tumor classification. But the real problem comes when segmentation of the tumor comes in to picture. The idea for tumor segmentation using deep learning became a reality when $\mathrm{CNN}$ are introduced. The ability of the $\mathrm{CNN}$ to find the features from the learning stage is commendable and one of the backbone of tumor segmentation technics.

The problem faced by most of the deep learning methods are the large database requirement for training. For classification of any other data images are available readily from cloud based services or public databases. Many such databases are available for free of cost. For example to classify the images of dogs from cats many images are available from internet for training the network. But for medical data the public databases are not available and hospitals are reluctant to reveal the patient details. This sometimes leads to inaccurate training results in deep learning. Data augmentation is performed by the algorithms to compensate with the database requirement in deep learning for medical images. GAN is another hot trending subject now. The ability of GAN to produce statistically similar image reduce the huge database requirement necessary for training the deep learning networks.

The automatic segmentation of tumor from MRI images has influenced many other MRI modalities like Magnetic Resonance Spectroscopy (MRS), Diffusion Tensor Imaging (DTI), and Perfusion Imaging (PI) which are used to segment the tumor using machine learning methods. The research in the area is still going on and will lead to better pre determination of tumor and treatment options.

\section{REFERENCES}

1. Pereira, Sérgio, et al. "Brain tumor segmentation using convolutional neural networks in MRI images." IEEE transactions on medical imaging 35.5 (2016): 12401251.

2. L. G. Nyul, J. K. Udupa, and X. Zhang, "New variants of a method ' of mri scale standardization," IEEE Transactions on Medical Imaging, vol. 19 , no. 2, pp. 143-150, 2000.

3. K.Umamaheswari ,P.Rajesh, S.Srinivasa Rao, P.Vinodh Babu. "Application of Segmentation Methodology for Extracting MRI Brain Tumor duly mitigating the Noise." 2015 International Conference on Computational Intelligence and Communication Networks

4. Malsawm Dawngliana, Daizy Deb, Mousum Handique, Sudipta Roy. "Automatic Brain Tumor Segmentation in MRI: Hybridized Multilevel Thresholding and Level Set." 2015 International Symposium on Advanced Computing and Communication (ISACC)

5. Deepthi Murthy T.S, G.Sadashivappa. "Brain Tumor Segmentation Using Thresholding, Morphological Operations And Extraction Of Features Of Tumor.” 2014 International Conference on Advances in Electronics, Computers and Communications (ICAECC).

6. Sanjivani Salwe, Ranjana Raut, Pratik Hajare. "Brain Tumor Pixels Detection using Adaptive Wavelet Based Histogram Thresholding and Fine Windowing." 2016 International Conference On Information Technology(InCITe)- The Next Generation IT Summit.

7. Parveen, Amritpal singh. "Detection of Brain Tumor in MRI Images, using Combination of Fuzzy C-Means and SVM." 2015 2nd International Conference on Signal Processing and Integrated Networks (SPIN).

8. Anupurba Nandi. "Detection of human brain tumour using MRI image segmentation and morphological operators.” 2015 IEEE International Conference on Computer Graphics, Vision and Information Security (CGVIS).

9. Hayder Saad Abdulbaqi, Mohd Zubir Mat Jafri, Kussay N. Mutter, Loay Kadom Abood, Iskandar Shahrim Bin Mustafa. "Segmentation and Estimation of Brain Tumor Volume in Computed Tomography Scan Images Using Hidden Markov Random Field Expectation Maximization Algorithm." 2015 IEEE Student Conference on Research and Development (SCOReD).
10. Amiya Halder, Anuva Pradhan, Sourjya Kumar Dutta and Pritam Bhattacharya. "Tumor Extraction from MRI images using Dynamic Genetic Algorithm based Image Segmentation and Morphological Operation.” International Conference on Communication and Signal Processing, April 6-8, 2016, India.

11. M. H. O. Rashid, M. A. Mamun, M. A. Hossain and M. P. Uddin "Brain Tumor Detection Using Anisotropic Filtering, SVM Classifier and Morphological Operation from MR Images." 2018 International Conference on Computer, Communication, Chemical, Material and Electronic Engineering (IC4ME2).

12. Reema Mathew A , Dr. Babu Anto P. "Tumor Detection And Classification Of Mri Brain Image Using Wavelet Transform And Svm." International Conference on Signal Processing and Communication (ICSPC'17) - 28th \& 29th July 2017.

13. Arun Kumar, Alaknanda Ashok, M.A.Ansari. "Brain Tumor Classification Using Hybrid Model Of PSO And SVM Classifier." International Conference on Advances in Computing, Communication Control and Networking (ICACCCN2018).

14. Bin Cui1, Mingchao Xie1, Chunxing Wang. “A Deep Convolutional Neural Network Learning Transfer to SVM-Based Segmentation Method for Brain Tumor.” 2019 IEEE 11th International Conference on Advanced Infocomm Technology.

15. Amiya Halder, Oyendrila Dobe. "Rough K-means and Support Vector Machine based Brain Tumor Detection." 2017 International Conference on Advances in Computing, Communications and Informatics (ICACCI).

16. Vijay Wasule, Poonam Sonar. "Classification of Brain MRI Using SVM and KNN Classifier." 2017 IEEE 3rd International Conference on Sensing, Signal Processing and Security (ICSSS).

17. Mohammad Havaei Pierre-Marc Jodoin, Hugo Larochelle. "Efficient interactive brain tumor segmentation as within-brain $\mathrm{kNN}$ classification." 2014 22nd International Conference on Pattern Recognition

18. I.Kalaivani, A.Sheryl Oliver, R.Pugalenthi. "Brain Tumor Segmentation Using Machine Learning Classifier.” 2019 Fifth International Conference on Science Technology Engineering and Mathematics (ICONSTEM).

19. Ketan Machhale, Hari Babu Nandpuru , Vivek Kapur , Laxmi Kosta. "MRI Brain Cancer Classification Using Hybrid Classifier (SVM-KNN)." 2015 International Conference on Industrial Instrumentation and Control (ICIC) College of Engineering Pune, India. May 28-30,2015.

20. B.Hachemi, S.Oudjemia, F.Alim, S. Seddiki, F.Talbi, M.Abdelaziz. "Cerebral Abnormalities Detection by RegionGrowing Segmentation and KNN Classification." Fifth international conference on Innovative Computing Technology (INTECH 2015).

21. M.Madheswaran, M.Madheswaran. "An Adroit Naïve Bayesian Based Sequence Mining Approach For Prediction Of Mri Brain Tumor Image." Fifth International Conference on Computing, Communications and Networking Technologies (ICCCNT).

22. D. Selvathi, Henry Selvaraj. "Segmentation Of Brain Tumor Tissues In Mr Images Using Multiresolution Transforms And Random Forest Classifier With Adaboost Technique." 2018 26th International Conference on Systems Engineering (ICSEng).

23. Najeebullah Shah, Sheikh Ziauddin, Ahmad R. Shahid. "Brain Tumor Segmentation and Classification using Cascaded Random Decision Forests." 2017 14th International Conference on Electrical Engineering/Electronics, Computer, Telecommunications and Information Technology (ECTI-CON).

24. Chao Ma, Gongning Luo, Student Member, IEEE, and Kuanquan Wang, Senior Member, IEEE. "Concatenated and Connected Random Forests With Multiscale Patch Driven Active Contour Model for Automated Brain Tumor Segmentation of MR Images." IEEE TRANSACTIONS ON MEDICAL IMAGING, VOL. 37, NO. 8, AUGUST 2018.

25. Dr.D.Saraswathi, B.Lakshmi Priya, R.Punitha Lakshmi. "Brain Tumor Segmentation and Classification using Self Organizing Map.” 2019 IEEE International Conference on System, Computation, Automation and Networking (ICSCAN).

26. Virupakshappa ; Basavaraj Amarapur. "An Automated Approach for Brain Tumor Identification using ANN Classifier." International Conference on Current Trends in Computer, Electrical, Electronics and Communication (ICCTCEEC-2017).

Published By:

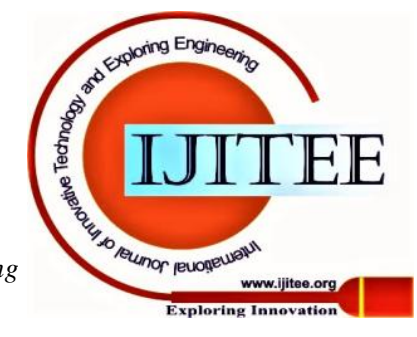


27. T.Chithambaram, K.Perumal. "Brain Tumor Segmentation using Genetic Algorithm and ANN Techniques." IEEE International Conference on Power, Control, Signals and Instrumentation Engineering (ICPCSI-2017).

28. Vinod Kumar, Jainy Sachdeva, Indra Gupta Niranjan Khandelwal, Chirag Kamal Ahuja. "Classification of brain tumors using PCA-ANN.” 011 World Congress on Information and Communication Technologies.

29. Aimin Yang 1, Xiaolei Yang, Wenrui W, Huixiang Liu1, And Yunxi Zhuansun. "Research on Feature Extraction of Tumor Image Based on Convolutional Neural Network." SPECIAL SECTION ON NEW TRENDS IN BRAIN SIGNAL PROCESSING AND ANALYSIS, IEEE Access.

30. Jia Liu , Fang Chen, Changcun Pan, Mingyu Zhu , Xinran Zhang, Liwei Zhang,and Hongen Liao, Senior Member, IEEE. "A Cascaded Deep Convolutional Neural Network for Joint Segmentation and Genotype Prediction of Brainstem Gliomas." IEEE TRANSACTIONS ON BIOMEDICAL ENGINEERING, VOL. 65, NO. 9, SEPTEMBER 2018.

31. Changhee Han, Leonardo Rundo, Ryosuke Araki, Yudai Nagano, Yujiro Furukawa, Giancarlo Mauri, Hideki Nakayama, Hideaki Hayashi. "Combining Noise-to-Image and Image-to-Image GANs: Brain MR Image Augmentation for Tumor Detection." 10.1109/ACCESS.2019.2947606, IEEE Access.

32. Kai Hu ; Qinghai Gan ; Yuan Zhang ; Shuhua Deng ; Fen Xiao ; Wei Huang ; Chunhong Cao ; Xieping Gao. "Brain Tumor Segmentation Using Multi-Cascaded Convolutional Neural Networks and Conditional Random Field." IEEE Access.

33. Mohammad Havaei, Axel Davy, David Warde-Farley. "Brain tumor segmentation with Deep Neural Networks." Medical Image AnalysisVolume 35, January 2017, Pages 18-31.

34. Xiaomei Zhao, Yihong Wu1, Guidong Song, Zhenye Li,Yong Fan, and Yazhuo Zhang. "A deep learning model integrating FCNNs and CRFs for brain tumor segmentation." Springer International Publishing AG 2016 A. Crimi et al. (Eds.): BrainLes 2016, LNCS 10154, pp. 75-87, 2016.

35. Konstantinos Kamnitsas, Christian Ledig, Virginia F.J. Newcombe, Joanna P. Simpson, Andrew D. Kane, David K. Menon, Danie Rueckert, Ben Glocker. "Efficient Multi-Scale 3D CNN with Fully Connected CRF for Accurate Brain Lesion Segmentation." Computer Vision and Pattern Recognition (cs.CV); Artificial Intelligence (cs.AI).

36. Huimin $\mathrm{Hu}$, Quanhua $\mathrm{Mu}$, Zhaoshi Bao,Xiaolong Fan, Jiguang Wang, Tao Jiang. "Mutational Landscape of Secondary Glioblastoma Guides MET-Targeted Trial in Brain Tumor." Hu et al., 2018, Cell 175, 1665-1678 November 29, $2018^{\text {a }} 2018$ Elsevier Inc.

37. Michal Drozdzal Gabriel Chartrand EugeneVorontsov Maha Shakeri. "Learning normalized inputs for iterative estimation in medical image segmentation." Medical Image Analysis Volume 44, February 2018 , Pages 1-13.

\section{AUTHORS PROFILE}

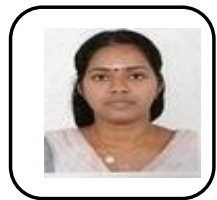

Aswani K received the master's degree from Anna University, Chennai, India, in 2013. She is currently pursuing the Ph.D. degree with Noorul Islam Center for Higher Education, India. Her research interests include computer vision and medical image analysis.

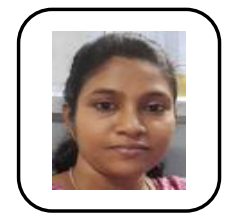

Dr. D. Menaka received her Ph. D. from Noorul Islam Center for Higher Education, India in 2018. His research interests include Deep Learning, Artificial Intelligence, image processing and Robotics

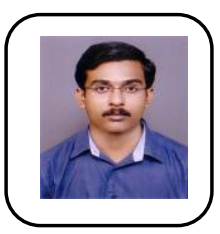

Manoj M K received the master's degree from Anna University, Chennai, India, in 2013. His research interests include Artificial Intelligence and image processing 